\title{
STONE-WEIERSTRASS THEOREMS FOR THE STRICT TOPOLOGY
}

\author{
CHRISTOPHER TODD
}

1. Let $X$ be a locally compact Hausdorff space, $E$ a (real) locally convex, complete, linear topological space, and $\left\langle C^{*}(X, E), \beta\right\rangle$ the locally convex linear space of all bounded continuous functions on $X$ to $E$ topologized with the strict topology $\beta$. When $E$ is the real numbers we denote $C^{*}(X, E)$ by $C^{*}(X)$ as usual. When $E$ is not the real numbers, $C^{*}(X, E)$ is not in general an algebra, but it is a module under multiplication by functions in $C^{*}(X)$.

This paper considers a Stone-Weierstrass theorem for $\left\langle C^{*}(X), \beta\right\rangle$, a generalization of the Stone-Weierstrass theorem for $\left\langle C^{*}(X, E), \beta\right\rangle$, and some of the immediate consequences of these theorems. In the second case (when $E$ is arbitrary) we replace the question of when a subalgebra generated by a subset $S$ of $C^{*}(X)$ is strictly dense in $C^{*}(X)$ by the corresponding question for a submodule generated by a subset $S$ of the $C^{*}(X)$-module $C^{*}(X, E)$. In what follows the symbols $C_{0}(X, E)$ and $C_{00}(X, E)$ will denote the subspaces of $C^{*}(X, E)$ consisting respectively of the set of all functions on $X$ to $E$ which vanish at infinity, and the set of all functions on $X$ to $E$ with compact support.

Recall that the strict topology is defined as follows [2]:

Definition. The strict topology $(\beta)$ is the locally convex topology defined on $C^{*}(X, E)$ by the seminorms

$$
\|f\|_{\phi, \nu}=\operatorname{Sup}_{x \in X}|\phi(x) f(x)|_{\nu}
$$

where $\nu$ ranges over the indexed topology on $E$ and $\phi$ ranges over $C_{0}(X)$.

When $E$ is the real numbers the above definition may be given simply as: a net $\left\{f_{\alpha}: \alpha \in A\right\}$ converges strictly to a function $f$ iff $\left\{\phi f_{\alpha}: \alpha \in A\right\}$ converges uniformly to $\phi f$ for each $\phi$ in $C_{0}(X)$. It is known that $C^{*}(X, E)$ is complete in the strict topology [2].

2. Strict approximation in $C^{*}(X)$. Stone-Weierstrass theorems were given for $C^{*}(X)$, under special restrictions by Buck [2]. The exact analogue of the classical theorem (for complex valued functions) was obtained by Glicksberg [3] as a corollary to a version of Bishop's generalized Stone-Weierstrass theorem [1]. The proof is not elementary. We give here a proof which follows quite simply from Buck's

Received by the editors May 1, 1964. 
original theorems. These theorems were proved under the additional hypothesis that either (a) the subalgebra in question contained a function vanishing nowhere or (b) the space $X$ on which the functions were defined was $\sigma$-compact.

Theorem 1. Let $X$ be a locally compact Hausdorff space and let $\mathfrak{A}$ be a strictly closed subalgebra of $C^{*}(X)$ which separates points of $X$ and which for each $x$ in $X$, contains a function $g$ with $g(x) \neq 0$, then $\mathfrak{A}=$ $C^{*}(X)$.

Proof. Let $f \in C^{*}(X)$ and let $\phi \in C_{0}(X)$ and $\epsilon>0$ be given. Let $K(\phi)$ be the $\sigma$-compact set outside of which $\phi$ vanishes identically. $K(\phi)$ may be replaced by a regularly $\sigma$-compact subset $K$ of $X$ (i.e. $K$ is a countable union of compact subsets $K_{n}$, with $K_{n}$ contained in the interior of $K_{n+1}$ ) which contains $K(\phi)$ and which is an open $F_{\sigma}$ set [2]. $K$ is a locally compact Hausdorff space in its relative topology and hence the strict topology is defined for $C^{*}(K)$, and $\phi$ is in $C_{0}(K)$. $\mathfrak{A}$ restricted to $K$ is dense in $C^{*}(K)$ in the strict topology by (b) above, consequently there is a function $g$ in $\mathfrak{A}$ such that $\mid \phi(x) f(x)$ $-\phi(x) g(x) \mid<\epsilon$ for $x$ in $K$. Since $\phi f$ and $\phi g$ vanish identically outside $K$, we have $\|g-f\|_{\phi}<\epsilon$. The conclusion now follows from the fact that $\left\langle C^{*}(X), \beta\right\rangle$ is complete.

The usual theorem for the complex case follows immediately from the above. We also note without proof the following obvious consequences of Theorem 1. (1) If $X$ is a product of locally compact Hausdorff spaces which is locally compact, then every function in $C^{*}(X)$ may be strictly approximated by finite sums of finite products of functions of one variable on $X$. (2) There is a 1-1 correspondence between the $\beta$-closed ideals of $C^{*}(X)$ and the closed subsets of $X$. In particular there is a $1-1$ correspondence between the maximal $\beta$-closed ideals and the points of $X$, and each $\beta$-closed ideal is the intersection of all $\beta$-closed maximal ideals which contain it.

Theorem 1 may be applied to obtain the following result.

Theorem 2. If $X$ is a locally compact and $\sigma$-compact Hausdorff space, then $\left\langle C^{*}(X), \beta\right\rangle$ is separable iff $X$ is metrizable.

Proof. Suppose that $X$ is metrizable, hence since $X$ is $\sigma$-compact, $X$ is separable and satisfies the second axiom of countability. Let $B$ be a countable base for the topology on $X$. For each $B$ in $B$, the complement of $B$, being closed, is the zero set of a function $f_{B}$ in $C^{*}(X)$. Let $\mathfrak{A}$ be the countable algebra generated by $\left\{f_{B} \mid B \in \mathbb{B}\right\}$ over the rationals. $\mathfrak{A}$ clearly satisfies the conditions of Theorem 1 and hence is $\beta$-dense in $C^{*}(X)$. Conversely let $S$ be a countable $\beta$-dense subset 
of $C^{*}(X)$, and let $\mathfrak{A}_{S}$ be the countable algebra generated by $S$ over the rationals. Let $\phi$ be any element of $C_{0}(X)$ which vanishes nowhere on $X$. Let $\phi \mathfrak{A}_{S}=\left\{\phi f \mid f \in \mathfrak{A}_{S}\right\} \subseteq C_{0}(X)$. By the classical Stone-Weierstrass theorem $\phi \mathfrak{H}_{S}$ is uniformly dense in $C_{0}(X)$. Let $\mathfrak{H}^{*}$ be the subset of $\phi \mathfrak{H}_{S}$ consisting of all functions $f$ such that $\|f\| \leqq 1$. These functions may be used to define a function $E$ on $X$ into the unit cube $Q^{w}$ $=\prod_{f}\left\{[0,1]_{f} \mid f \in \mathfrak{A}^{*}\right\}$ by $E(x)_{f}=f(x) . E$ is easily shown to be a homeomorphism, hence $X$ is metrizable.

It is conjectured that separability for $\left\langle C^{*}(X), \beta\right\rangle$ under the sole hypothesis that $X$ is locally compact and Hausdorff is characterized by the statement: $\left\langle C^{*}(X), \beta\right\rangle$ is separable iff $X$ is metrizable and separable. Note that if $X$ is metrizable and separable then $X$ is necessarily $\sigma$-compact.

3. Strict approximation in $C^{*}(X, E)$. Let $S$ be a subset of $C^{*}(X, E)$ and let $x$ be an element of $X, M$ a closed subspace of $E$. If $S(x)$ $=\{\alpha \in E \mid \alpha=f(x)$ for some $f \in S\}$ is contained in $M$ then we will say $S$ is restricted to $M$ at $x$. We will call a subset $S$ of $C^{*}(X, E)$ unrestricted if for each maximal closed subspace $M$ of $E$ and each point $x$ of $X$, there is a function $f$ in $S$ with $f(x) \notin M$.

Theorem 3. Let $X$ be a locally compact Hausdorff space, and $E$ a locally convex, complete, linear topological space. If $S$ is an unrestricted submodule of $C^{*}(X, E)$, then $S$ is strictly dense in $C^{*}(X, E)$.

We first give a proof of the following fact due to Buck [2].

Lemma. $C_{00}(X, E)$ is strictly dense in $C^{*}(X, E)$.

Proof. Let $f \in C^{*}(X, E), \phi \in C_{0}(X)$, a seminorm $\nu$ for $E$ and $\epsilon>0$ be given. $\phi f$ is in $C_{0}(X, E)$. Let $K \subseteq X$ be the compact set outside of which $|\phi(x) f(x)|_{\nu}<\epsilon / 2$. There exists a function $\theta$ in $C_{00}(X)$ such that $\theta(x)=1$ for $x$ in $K$ and $\|\theta\| \leqq 1 . \theta f$ is in $C_{00}(X, E)$ and $|\phi \theta f(x)-\phi f(x)|$. $<\epsilon$ for every $x$ in $X$. Therefore $f$ is in the strict closure of $S$.

Proof of Theorem 3. In view of the preceding lemma we need only show that if $S$ is an unrestricted submodule, then $C_{00}(X, E)$ lies within the strict closure of $S$. We show in fact that $C_{00}(X, E)$ is in the uniform closure of $S$ (it is true that $C_{0}(X, E)$ is in the uniform closure of $S$ ).

Let $f$ be any function in $C_{00}(X, E)$ and let a seminorm $\rho$ for $E$ and $\epsilon>0$ be given. Let $\Psi_{\rho}(x)=|f(x)|_{\rho} . \Psi_{\rho}$ is in $C_{00}(X)$. Let $K$ be the compact set outside of which $\Psi_{\rho}$ vanishes identically. For each $g$ in $S, \Psi_{\rho} g$ is in $S$ and $\Psi_{\rho} g(x)=0$ for $x$ not in $K$. Let $x_{0}$ be any point of $K$ and suppose $f\left(x_{0}\right)=\xi$. By hypothesis there is a function $g$ in $S$ such that $g\left(x_{0}\right)=\xi$. Let $\Psi_{\rho}\left(x_{0}\right)=a$. If $a \neq 0$ let $h_{x_{0}}$ be the function $a^{-1} \Psi_{\rho} g . h_{x_{0}}\left(x_{0}\right)$ 
$=\xi, h_{x_{0}}(x)=0$ for $x$ not in $K$, and $h_{x_{0}}$ is in $S$. Thus there exists a neighborhood $U_{x_{0}}$ of $x_{0}$ such that $\left|h_{x_{0}}(y)-f(y)\right|_{\rho}<\epsilon$ for $y$ in $U_{x_{0}}$. Suppose that $\Psi_{\rho}\left(x_{0}\right)=0$. Then let $h_{x_{0}}=\Psi_{\rho} g .\left|h_{x_{0}}\left(x_{0}\right)-f\left(x_{0}\right)\right|_{\rho}=\left|f\left(x_{0}\right)\right|_{\rho}=0$ and again by the continuity of seminorms we may choose a neighborhood $U_{x_{0}}$ of $x_{0}$ with $\left|h_{x_{0}}(y)-f(y)\right|_{\rho}<\epsilon$ for $y$ in $U_{x_{0}}$. In the usual manner we obtain a finite number of points $x_{i}$ in $K$, of functions $h_{i}$ in $S$, and neighborhoods $U_{i}$ of $x_{i}$ such that if $y$ is in $K$ then $y$ is in $U_{i}$ for some $i$ and $\left|h_{i}(y)-f(y)\right|_{\rho}<\epsilon$. The set of neighborhoods $U_{i}$ is a point finite open cover of $K$ in its relative topology. Since $K$ is completely regular and compact, there exist functions $\phi_{i}$ in $C^{*}(K)=C(K)$ such that $\phi_{i}\left(U_{i}\right) \subset[0,1], \phi_{i}$ vanishes identically in $K$ outside $U_{i} \cap K$, and $\sum_{i} \phi_{i}(x)=1$ for each $x$ in $K$. Since $K$ is a compact subset of a completely regular space each $\phi_{i}$ may be extended to a function in $C^{*}(X)$, so that the functions $\phi_{i} h_{i}$ are defined for each $i$ and are in $S$. The function $F=\sum_{i} \phi_{i} h_{i}$ is in $S$, is identically 0 outside $K$ and $\|F-f\|_{\rho}<\epsilon$. Thus $C_{00}(X, E)$ lies in the uniform closure of $S$. From the preceding lemma it follows that $S$ is strictly dense in $C^{*}(X, E)$.

Corollary 1. Any subspace of $C^{*}(X, E)$ which is a $C_{00}(X)$ submodule is strictly dense in $C^{*}(X, E)$. A strictly closed $C_{00}(X)$ submodule is a $C^{*}(X)$ module.

Corollary 2. A function $f$ in $C^{*}(X, E)$ is in the strict closure of the $C^{*}(X)$ submodule generated by an arbitrary subset $S$ of $C^{*}(X, E)$ if and only if for any $x$ in $X$ and maximal closed subspace $M$ of $E, S(x) \subseteq M$ implies $f(x) \in M$.

Theorem 3 generalizes results obtained in [2]. We use the above results to characterize the strictly closed maximal submodules of $C^{*}(X, E)$.

If $M$ is a maximal closed subspace of $E$ and $x$ is a fixed point of $X$, let $S_{x, M}=\left\{f \in C^{*}(X, E) \mid f(x) \in M\right\}$. Let $y$ be any point of $X$ with $y \neq x$, and $a \in E, b \in M$. Let $\phi_{1}$ and $\phi_{2}$ be functions in $C^{*}(X)$ such that $\phi_{1}(y)=1, \phi_{1}(x)=0$, and $\phi_{2}(y)=0, \phi_{2}(x)=1$. Let $h=a \phi_{1}+b \phi_{2} . h(x)=b$, $h(y)=a$ and $h$ is in $S_{x, M}$. Thus $S_{x, M}(x)=M$ and $S_{x, M}(y)=E$ for each $y$ in $X$ with $y \neq x$. By Corollary $2, S_{x, M}$ is a strictly closed submodule of $C^{*}(X, E)$. We show that it is a maximal submodule. Suppose that $S^{\prime}$ is a submodule of $C^{*}(X, E)$ such that $S^{\prime} \supset S_{x, M}, S^{\prime} \neq S_{x, M}$. Then there is a function $f$ in $S^{\prime}$ such that $f(x) \notin M$. Let $f(x)=\lambda \in E$. $E$ may be represented as a direct sum $M \oplus N$ where $N$ is a complement of $M$. Then for each $\xi \in E, \xi=m+n$, with $m \in M, n \in N$, and so for some $m_{0}$ in $M$ and $n_{0}$ in $N, \lambda=m_{0}+n_{0}$. There exists a function $g$ in $S_{x, M}$ such that $g(x)=m_{0}$. The function $f^{\prime}=f-g$ in $S^{\prime}$ has the property $f^{\prime}(x)=n_{0}$. 
Since $M$ is of deficiency 1 in $E$ each point of $N$ is of the form $\alpha n_{0}$, where $\alpha$ is a real number. Thus if $\xi$ is any element of $E, \xi=m+\alpha n_{0}$, there is a function $h$ in $S^{\prime}, h=g+\alpha f^{\prime}$ (where $g$ is a function in $S_{x, M}$ ) such that $h(x)=\xi$, hence $S^{\prime}(x)=E$. Also $S^{\prime}(y)=E$ for any point $y$ in $X$. Now let $j$ be any function in $C^{*}(X, E)$ and let $j(x)=\eta$. There is a function $g_{1}$ in $S^{\prime}$ with $g_{1}(x)=\eta$. Let $g_{2}=j-g_{1} \cdot g_{2}(x)=0$ so $g_{2}$ is in $S_{x, M} \subset S^{\prime} . j=g_{1}+g_{2}$ so $j$ is in $S^{\prime}$ thus $S^{\prime}$ is $C^{*}(X, E)$. Consequently $S_{x, M}$ is a strictly closed maximal submodule. Conversely it is easy to see that if $S$ is a strictly closed maximal submodule of $C^{*}(X, E)$ then $S$ is of the form $S_{x, M}$.

Note that unlike the situation for maximal ideals there may exist maximal submodules which are not strictly closed. Let $E$ be any space (with the usual requirements) which admits a discontinuous linear functional $L$. Let $N$ be the null space of $L . N$ is a maximal subspace of $E$ and is dense in $E$. Let $x$ be any point of $X$ and let $S$ be the set of all functions $f$ in $C^{*}(X, E)$ such that $f(x) \in N$. It is a routine verification that $S$ is a $C^{*}(X)$ submodule of $C^{*}(X, E)$. We show that $S$ is a maximal submodule. Let $\eta$ be any element of $E$ with $\eta$ not in $N$ and let $f$ be a function in $C^{*}(X, E)$ with $f(x)=\eta$. If $\xi$ is any point of $E$ then $\xi=\alpha \eta+n$ with $n \in N$ since $N$ is maximal. Let $g$ be any function in $C^{*}(X, E)$ and let $g(x)=\lambda=\partial \eta+n, n \in N$. Let $g_{1}=g-\partial f . g_{1}(x)$ $=g(x)-\partial f(x)=n$ so $g_{1}$ is in $S . g=g_{1}+\partial f$ so $S$ is a maximal submodule. $S$ is not strictly closed, however, by Corollary 2 of Theorem 3 . We state the preceding facts as

Theorem 4. Let $X$ be a locally compact Hausdorff space, E a locally convex, complete linear space. The strictly closed maximal submodules of $C^{*}(X, E)$ are the submodules of the form $S_{x, M}$. If $E$ admits no discontinuous linear functional then every maximal submodule is closed.

TheORem 5. Let $X$ be a locally compact Hausdorff space and $E$ a locally convex, complete linear space. A strictly closed submodule $S$ of $C^{*}(X, E)$ is.the intersection of all the maximal strictly closed submodules that contain it.

Proof. Let $S$ be any strictly closed submodule of $C^{*}(X, E)$. Fix $x$ in $X$ and let $M_{x}$ be the closure of $S(x)$ in $E$. Let $\mathscr{T}(x, S)$ be the set of all maximal closed subspaces $M$ of $E$ which contain $M_{x} . M_{x}$ $=\cap\{M \mid M \in \mathscr{M}(x, S)\}$ by the Hahn-Banach theorem for locally convex spaces. Clearly for each $x$ in $X, S$ is contained in $S_{x, M}$ when $M$ is in $\mathfrak{N}(x, S)$. Let $S^{\prime}=\cap\left\{S_{x, M} \mid x \in X, M \in \mathfrak{N}(x, S)\right\} . S^{\prime}$ is a strictly closed submodule of $C^{*}(X, E)$ which contains $S$ and by Corollary 2 of Theorem 3 each function $f$ in $S^{\prime}$ is in $S$ so that $S^{\prime}=S$. 


\section{REFERENCES}

1. E. Bishop, A generalization of the Stone-Weierstrass theorem, Pacific J. Math. 11 (1961), 777-783.

2. R. C. Buck, Bounded continuous functions on a locally compact space, Michigan Math. J. 5 (1958), 95-104.

3. I. Glicksberg, Bishop's generalized Stone-Weierstrass theorem for the strict topology, Proc. Amer. Math. Soc. 14 (1963), 329-333.

UNIVERSITY OF WISCONSIN AND

UNIVERSITY OF CONNECTICUT

\section{WEAK LIMITS OF POWERS OF A CONTRACTION IN HILBERT SPACE ${ }^{1}$}

S. R. FOGUEL

Let $T$ be an operator, on the Hilbert space $H$, with $\|T\| \leqq 1$. Let

$$
H_{0}=\left\{x \mid \text { weak } \lim T^{n} x=0\right\}, \quad H_{1}=H_{0}^{\perp} .
$$

We shall use the facts, proved in [1], that

1. $x \in H_{0}$ if and only if $\lim \left(T^{n} x, x\right)=0$ (Theorem 3.1).

2. On $H_{1}$ the operator $T$ is unitary (Theorem 1.1).

Given $x \in H$ let $x=x_{0}+x_{1}$, where $x_{0} \in H_{0}$ and $x_{1} \in H_{1}$. The purpose of this note is to find conditions, on the sequence $\left(T^{n} x, x\right)$, that will imply that $x_{1}$ is generated by eigenvectors of $T$. This is related to the notion of mixing for ergodic transformations.

THEOREM 1. Let $y$ be in the subspace generated by $T^{n} x, T^{*_{n}} x$, $n=1,2, \cdots$. If $\lim \left(T^{n} y, x\right)=0$, then weak $\lim T^{n} y=0$.

Proof. Since $H_{0}$ and $H_{1}$ are invariant under $T$, it is enough to prove the theorem for the case when $x \in H_{1}$ (and thus also $y \in H_{1}$ ). If $n_{i}$ is any subsequence of the integers and $z=$ weak $\lim T^{n i y}$, then $z$ is orthogonal to $T^{k} x, k=0, \pm 1, \pm 2, \cdots$ (since $T$ is unitary on $H_{1}$ ). But $z$ belongs to the subspace generated by $T^{ \pm n} x$. Hence, $z=0$ and, thus, weak $\lim T^{n} y=0$.

Corollary. Let $P(\lambda)$ be a polynomial whose roots of modulus one are $\lambda_{1}, \cdots, \lambda_{k}$. If $\lim \left(T^{n} P(T) x, x\right)=0$, then $x=x_{0}+x_{1}$ where: $x_{0} \in H_{0}$, $x_{1}=\sum_{i=1}^{k} z_{i}, T z_{i}=\lambda_{i} z_{i}$.

Proof. Let $x=x_{0}+x_{1}$, where $x_{0} \in H_{0}$ and $x_{1} \in H_{1}$. By Theorem 1,

Received by the editors March 25, 1964.

1 This work was partially supported by NSF Grants GP87 and G14736 at Northwestern University. 